\title{
Empathy is Central to Entrepreneurship
}

\section{Yasuhiro (Yasu) Yamakawa (Babson College) \\ Brett Smith (Miami University of Ohio)}

\author{
KEYWORDS: Entrepreneurship, Innovation, \\ Management.
}

Successful entrepreneurs not only identify problems that must be solved, but also empathize with the people affected by them. In this discussion, Babson College's Yasu Yamakawa and Brett Smith of Miami University of Ohio propose that empathy is a quality that can be acquired only by going out into the world without preconceived notions, and being willing to "do and learn" rather than "learn and do." The biggest drawback to empathy: thinking too much. 